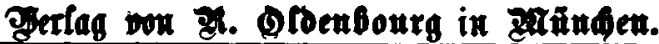

\section{Die Raturfräfte.}

Eine



geder 3aand koftet brofaint 3 ogark.

" " gefunt. 4 Dgark.

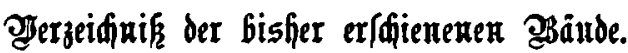

I. Band. Die SeGre vout Sqaar. Bemeinfafitide Dar-


f由nitten. 3roeite Auflage.

II. Band. Staft und Garbe. Eine gemeinfaj̆lide Dar= ftellurg ber Dptit. Bon Prof. Dr. Fr. 9ol. Pisto in פiett. (Doppelband.) 37 Bogen Teţt mit 148 \olzimn. 3 weite 2uflage.

III. Band. Dte Earme. RaA bem Frangöifíden bes Brof. Eajin in Parią beut/两 bearbeitet. Derausgegeben ourd Prof. Dr. Bhil. Corl in Pilnden. 19 Bogen Tent mit 92 5olf. innitten and eiter forbenbruttafel. Breite Iuflage.

IV. Band. Das Eaffer. BBon Brof. Dr. Pfaff in Erlangen, mit 21 Bogen Tegt unb 57 meift größeren \$olzidnitten.

V. Gand. Sotmmet und Erbe. Fine gemeinfaslide Bes f Bogen Tert mit 45 5olzimnitten unb 5 Tafeln.

VI. Band. Die electrif(ien Zlaturledfte. Der Mlag= netisurs, bie Electricität, bergaloanifhe Strom.

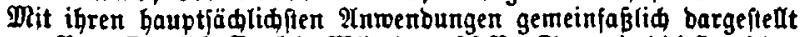



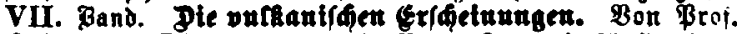

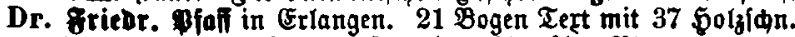

VIII. unb IX. Gand. Aus ber gurgetf. Bilber aus ber

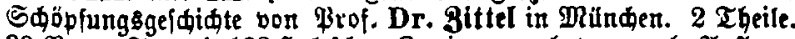
39 Bogen Tert mit 183 Folzidn. 3weite vermehrte u. vetb. Xuflage.

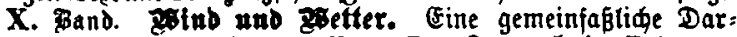
ftelung ber Meteorologie von \$rof. Dr. Eommel in Erlangen. 25 Bogen Text mit 66 Solzidmittent.

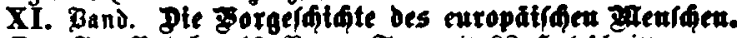
Bon Dr. F̈r. Makel. 19 Bogen Tert mit 92 Solzidnitten. 


\section{Broipectus.}

XII. Band. Zfau nnd Sebea bex Z゙fianzen. Bon Dr.

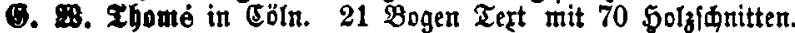

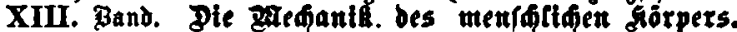

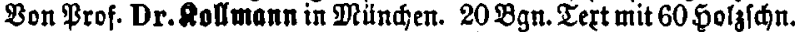


Brof. Dr. Fr. Miertel in Moplod. 20 Bogen Tert mit 132 Jorziकn.

XV. Band. Bas Spektrum und bie Spektralanainje. Bon Dr. \$. Beథ, Prof. Der Phgrt am Polptednitum in Stutt.

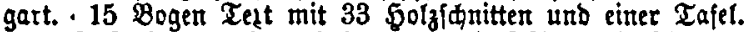

XVI. Band. Darwintsmus utio Egterprobukfton. Bon Prof. Dr. (E. E. M. Eartmann. 19 Bgn. Tert mit 46 5olgífnitten.

XVII. Band. Zels und Erbboben. Bon 5ofrath, Brof. Dr. Zerdinand Eenft. 26 Bogen. Tert mit 17 5olgldnitten.

XVIII. Gand. Ge/unbliettsfegre bes metr/arificn sorpers. Bon Dr. P. Niemeyer in Reipzig. 19 Bogen Tert mit 31 Jुulgldn.

XIX. Gand. Dte Ernährung des žlenldien. Bon Dr. Joljannes Mante in Viündjett. 26 Bogen Tert unt eine Photonraphie von $\mathfrak{3} . \mathfrak{v}$. Liebig.

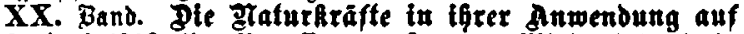


Witen. 22 Bogen Tert mit 64 5ूoljifnitten.

XXI. Band. Organtsmus ber ôn/ekten. Bon Profeīor

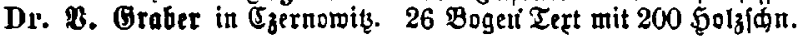

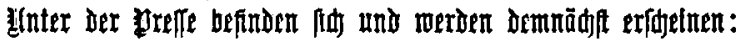

XXII. Gand. Seben ber sufefter. Brof. Dr. B. Graber in Ezernowit.


Bon Minifterialrath, Brof. Dr. (G. Mlayr in Münden.

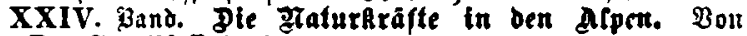
Prof. Dr. J̈r. Pfafi in Erlanger.

In borbereitung tefinden fit folgende gä̈nde:

Pollinger, Prof. Dr. in Mïnder. Die Zrrafien ber Epibemlen. Orejeld, Bribatbocent Dr. in Berlin. Zsacterten und żlfse.

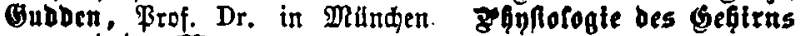
und bet Zerven.

Geller, Prof. Dr. in Siel. Die zarafiten.

3äger, Prof. Dr. Buft. in Etuttgart. Die nemfdilific Bar. Beitskraft.

3mmermann, Brof. Dr. in Bafel. Gcrunbbeif anb Sraukbett. \&rebs, Dr. in Frantfurt. Jite Eschfefwirkung oer atatur. Erảfte.

Iotenj, Dr., Minifterialrath in Bielt. Der Esald, ber żoden unb bas siltma. 


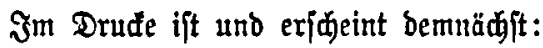

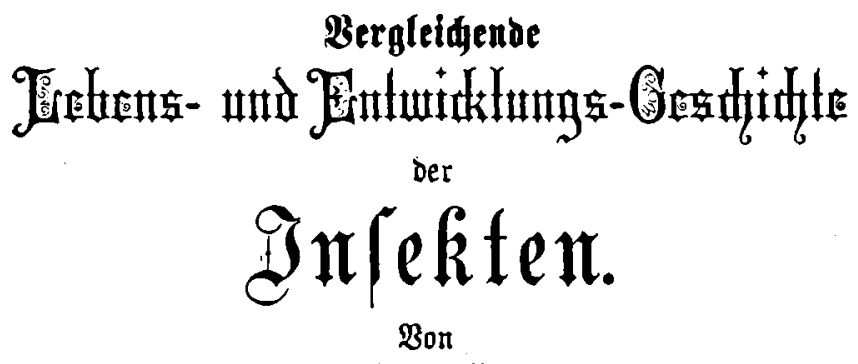

Dr. Bitus Graber.

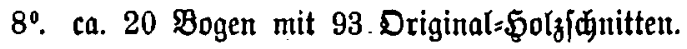

Preis ordiuär 3 Mart.

(II. Thyeil beş vorliegenden $\mathfrak{B e r l e z s . )}$

Ferner:

Die



im

\section{Gefeffed aftsegen \\ bolt}

Dr. Gearg Mayr,

P. b. Minifterialrath unb univerfttätsprofeffor, Borftanb bes fatufitifen Butreau's für bas sionigrei申 Bayern.

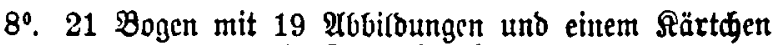
in Farbenbrut.

Preis oroinär 3 Mart. 
Im Drữe ift und eriđeint bemnäđft:

$$
\begin{aligned}
& \mathfrak{d} \mathfrak{i} \mathfrak{e} \mathfrak{d} \mathfrak{a} \mathfrak{t} \mathfrak{a} \mathfrak{k} \mathfrak{x} \mathfrak{a} \mathfrak{f} \mathfrak{t} \mathfrak{e} \\
& \text { in ben } \\
& \text { Pifpent. }
\end{aligned}
$$

\section{Dr. Jrriebrid Pfaff,}

D. B. Profefior an ber Iniberităt cexlangen.

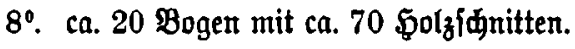

Preiả ordinär 3 Mark. 


\section{$\mathfrak{d} \mathfrak{a} \mathfrak{t} \mathfrak{a} \mathfrak{r} \mathfrak{k} \mathfrak{x} \mathfrak{a} \mathfrak{f} \mathfrak{f} \mathfrak{e}$}

Einundzranzagffer Band. 

Die



Son

\section{Dr. כुtitus (Graber,}

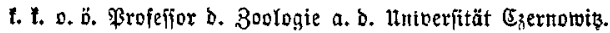

\section{Egheif.}

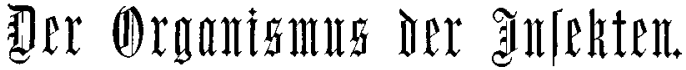

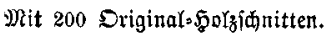

\section{Mündıen.}

Drut at Berlag bon $R$. Sibentourg. 1877. 
Heberfegurggredt vorbebalter. 